\title{
On the origin of visible photoluminescence in nanometer-size Ge crystallites
}

\author{
Yoshihiko Kanemitsu, Hiroshi Uto, and Yasuaki Masumoto \\ Institute of Physics, University of Tsukuba, Tsukuba, Ibaraki 305, Japan \\ Yoshihito Maeda \\ Hitachi Research Laboratory, Hitachi Ltd., Hitachi, Ibaraki 319-12, Japan
}

(Received 13 May 1992; accepted for publication 19 August 1992)

\begin{abstract}
We have studied the origin of visible photoluminescence of $\mathrm{Ge}$ nanocrystals in $\mathrm{SiO}_{2}$ glassy matrix. Spectroscopic analyses of Ge nanocrystals indicate that the room-temperature photoluminescence comes from Ge nanocrystals of diameter of $4 \mathrm{~nm}$ or less. High-resolution electron microscopic studies imply that the structure of Ge nanocrystals of diameter $\leqslant 4 \mathrm{~nm}$ differs from the diamond structure. These data suggest that new nanostructure crystalline $\mathrm{Ge}$ having a character of direct optical transition exhibits the visible photoluminescence.
\end{abstract}

Optical and electronic properties of semiconductor nanocrystallites, often called quantum dots, have attracted much attention, because they exhibit new quantum phenomena and have potentials for becoming novel and future photonic devices. ${ }^{1}$ Most of the previous works have been studied on nanocrystals or quantum dots made from direct-gap semiconductors such as $\mathrm{CdS}, \mathrm{CdSe}, \mathrm{CuCl}$, etc., experimentally ${ }^{1}$ and theoretically. ${ }^{2}$ Very recently, optical properties of nanostructures made from indirect-gap semiconductors such $\mathrm{Si}^{3,4} \mathrm{Ge}^{5}$ etc. have been reported. The discovery of luminescence in nanocrystals of $\mathrm{Si}$ and $\mathrm{Ge}$ is an extremely important scientific breakthrough with enormous technological implications, since it opens a new possibility for group IV semiconductors as new materials for optoelectronic applications. The origin and mechanism of strong visible luminescence in $\mathrm{Si}$ and Ge nanocrystals at room temperature are currently under discussion. ${ }^{6}$ In this letter, we present an origin of strong visible photoluminescence of $\mathrm{Ge}$ nanocrystals in $\mathrm{SiO}_{2}$ glassy matrix. Spectroscopic and electron microscopic studies show that a new Ge nanostructure of diameter $\leqslant 4 \mathrm{~nm}$ in $\mathrm{SiO}_{2}$ glassy matrix exhibits the strong room-temperature photoluminescence.

The samples were prepared by a method of rfmagnetron cosputtering of $\mathrm{Ge}$ and $\mathrm{SiO}_{2}$, as previously reported. $^{5}$ The Ar pressure and the rf power were $3 \times 10^{-3}$ Torr and $1.2 \mathrm{~kW}$, respectively. Thin films of the mixture of Ge and $\mathrm{SiO}_{2}$ were deposited onto $\mathrm{Si}$ substrates, and then annealed in an Ar gas atmosphere for $30 \mathrm{~min}$ at 300,600, or $800{ }^{\circ} \mathrm{C}$ in order to grow $\mathrm{Ge}$ nanocrystals in $\mathrm{SiO}_{2}$ glassy matrix and to control the size of $\mathrm{Ge}$ nanocrystals. The concentration of chemical elements except $\mathrm{Ge}, \mathrm{Si}$, and $\mathrm{O}$ were less than $0.1 \mathrm{ppm}$, which were measured by an inductively coupled plasma emission spectroscopy. The crystallinity and size of $\mathrm{Ge}$ nanocrystals in $\mathrm{SiO}_{2}$ matrix were studied by using a high-resolution electron microscopy (HREM).

The photoluminescence (PL) spectra of Ge nanocrystallites were measured by using 454.5-514.5 nm excitation light from an Ar ion laser. The calibration for the spectral sensitivity of the measuring system was performed by using a tungsten standard lamp. The temperature was varied from 10 to $300 \mathrm{~K}$ by controlling the flow rate of cold gas of
$\mathrm{He}$ in a cryostat. Excitation spectra of PL at the peak photon energy were measured by using a Xe lamp and a monochromator. Picosecond PL decay under a 200 ps and $514.5 \mathrm{~nm}$ laser excitation was measured using a monochromator of subtractive dispersion and a synchroscan streak camera.

The size distribution of Ge nanocrystals in samples used in this work are shown in Fig. 1. The size distribution of Ge nanocrystals in two samples (solid lines in Nos. 2 and 3 ) is characterized by a log-normal function given in Ref. 7. In samples (Nos. 2 and 3), the volume-average diameter $d_{v}$ is calculated using the log-normal function. However, in the sample No. 1, the size distribution of Ge nanocrystals is highly asymmetric and $d_{v}$ is directly evaluated from the size distribution of Ge nanocrystals in Fig. 1. Sample parameters are summarized in Table I.

Figure 2 shows typical HRFM images of Ge nanocrystals in $\mathrm{SiO}_{2}$ glassy matrix. In $\mathrm{Ge}$ nanocrystals larger than $5 \mathrm{~nm}$ in diameter, the interplane spacing is $0.326 \mathrm{~nm}$ and the surface of the Ge crystals consists of the (111) planes of the diamond structure. However, in Ge nanocrystals of diameter $\leqslant 4 \mathrm{~nm}$, the spacing is $0.298 \mathrm{~nm}$ and this spacing cannot be derived from the diamond structure

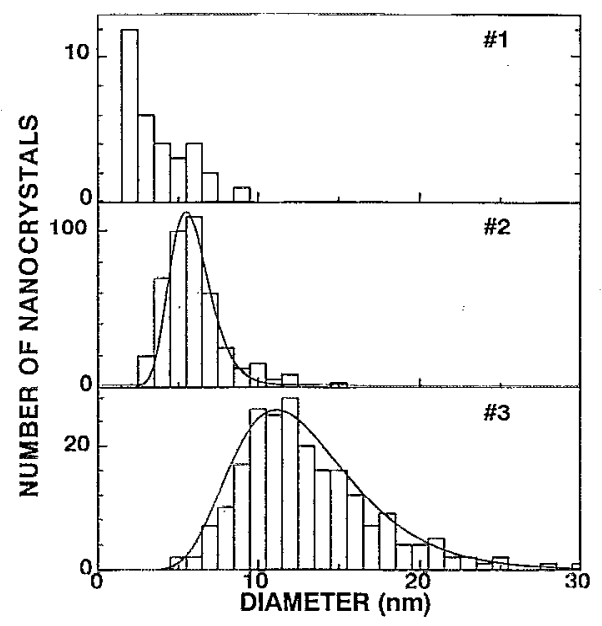

FIG. 1. The size distribution of Ge nanocrystals in $\mathrm{SiO}_{2}$ matrix. The solid lines are log-normal functions. 
TABLE I. Sample parameters including the annealing temperature $T_{A}$, the volume-average diameter $d_{\iota}$, the PL peak energy $E_{p}$ the volume fraction of Ge nanocrystals with diameter $<4 \mathrm{~nm} V_{4}$, the PL intensity $I_{\mathrm{TL}}$, and the time constant of the PL decay $\tau$.

\begin{tabular}{lcccccc}
\hline \hline Sample No. & $\begin{array}{c}T_{A} \\
{ }^{\circ} \mathrm{C}\end{array}$ & $\begin{array}{c}d_{v} \\
(\mathrm{~nm})\end{array}$ & $\begin{array}{c}E_{p} \\
(\mathrm{eV})\end{array}$ & $V_{4}^{\mathrm{a}}$ & $I_{\mathrm{pL}}{ }^{\mathrm{a}}$ & $\begin{array}{c}{ }^{T} \\
(\mathrm{~ns})\end{array}$ \\
\hline 1 & 300 & 4.2 & 2.3 & 100 & 100 & 0.85 \\
2 & 600 & 6.0 & 2.2 & 9.8 & 7.6 & 0.90 \\
3 & 800 & 14.4 & 2.3 & $\leq 0.1$ & $0.8^{\mathrm{b}}$ & 0.86 \\
\hline \hline
\end{tabular}

${ }^{8}$ Normalized values.

${ }^{\mathrm{t}}$ The PL intensity of the sample No. 3 is comparable to that of the as-deposited sample.

of bulk Ge. This fact implies that the structure of nanocrystalline Ge changes critically at the size of about $4 \mathrm{~nm}$. The volume fraction of $\mathrm{Ge}$ crystals of diameter $\leqslant 4 \mathrm{~nm}$ to total Ge crystals, $V_{4}$ are shown in Table I. This volume fraction, $V_{4}$, is a very important parameter in the understanding of the origin of the visible photoluminescence from $\mathrm{Ge}$ nanocrystals.

Figure 3 shows the PL spectra from Ge nanocrystals at room temperature under $488 \mathrm{~nm}$ laser excitation and the excitation spectra of the PL peak. Broad PL spectra exhibiting the peak of 2.2-2.3 eV were observed in all samples. The quantum efficiency as high as about $0.5 \%$ was obtained in the sample No. 1 and the relative PL intensity in three samples are summarized in Table I. The peak energy of the PL spectrum scarcely depends on the average diameter of Ge nanocrystals in the sample, $d_{v}$. The peak energy of the excitation spectrum near $530 \mathrm{~nm}$ also does not depend on the sample and the excitation spectrum differs from the absorption spectrum of bulk $\mathrm{Ge}$ of the diamond structure. However, it was found that the PL intensity of the sample is proportional to the volume fraction $V_{4}$. Therefore, it is concluded that Ge nanocrystals in the $\leqslant 4$ nm size regime exhibit the strong visible photoluminescence.

The PL decay at the peak photon energy was approximately described as a single exponential having a time

(a)

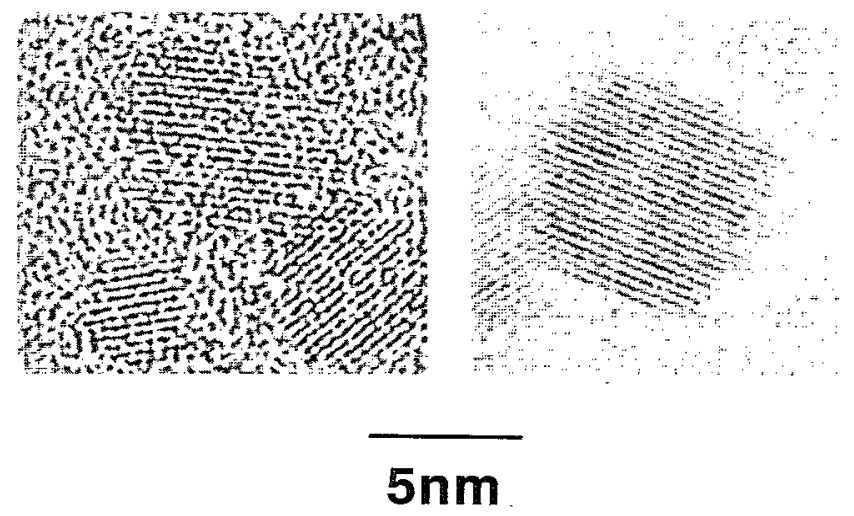

FIG. 2. HREM images of Ge nanocrystals with the diameter of (a) $<4$ $\mathrm{nm}$ and (b) $6.7 \mathrm{~nm}$. The spacing in Ge nanocrystals of diameter $\leqslant 4 \mathrm{~nm}$ is $0.298 \mathrm{~nm}$. The spacing of the larger nanocrystals is $0.326 \mathrm{~nm}$.

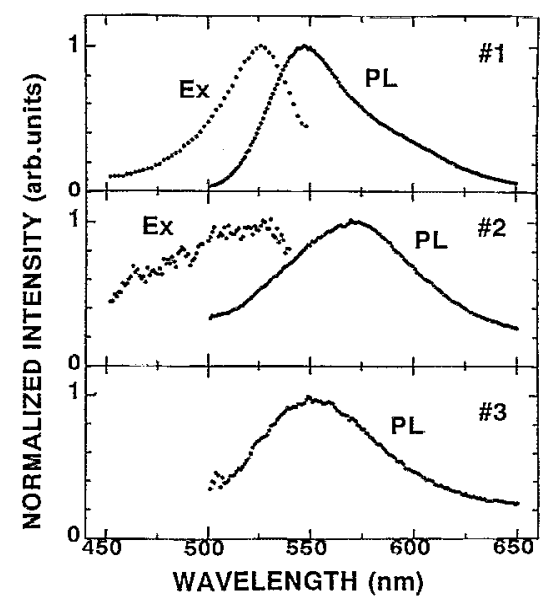

FIG. 3. PL spectra under $488 \mathrm{~nm}$ laser excitation and excitation spectra at the peak of the PL spectrum in three different samples.

constant $\tau$ and $\tau$ is summarized in Table $\mathrm{I}$. The PL lifetime $\tau$ does not depend on the photon energy in the broad PL band. No temperature dependence of $\tau$ was observed between 10 and $300 \mathrm{~K}$. Since $\tau$ is about $40 \mu$ s near $4 \mathrm{~K}$ in bulk Ge, ${ }^{8}$ the PL decay rate is enhanced in Ge nanocrystals. Table I shows that $\tau$ does not depend on the average diameter of Ge nanocrystals. The fast PL decay implies that the indirect-gap semiconductor $\mathrm{Gc}$ begins to have a character of direct optical transition as size decreases.

Figure 4 shows the temperature dependence of PL intensity and spectrum in the sample No. 1. The PL spectrum does not depend on the temperature, but the PL intensity, $I_{\mathrm{PL}}$, slightly depends on the temperature in all samples. If the radiative transition is excitonic and controls $\tau$ and $I_{\mathrm{PL}}$, the temperature dependence of $I_{\mathrm{PL}}$ in the $\ln I_{\mathrm{PL}}$ $-1 / \mathrm{T}$ plot gives the dissociation energy (or binding en-

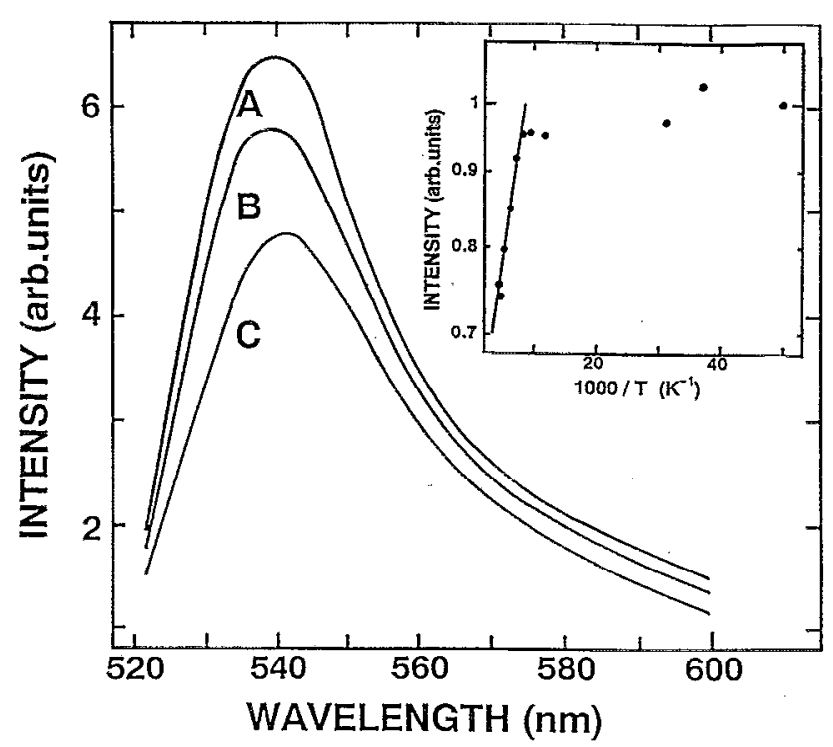

FIG. 4. Temperature dependence of the PL spectrum in the sample No. 1:A;18 K, B;85 K, and C;230 K. The inset shows the temperature dependence of the PL intensity at the peak. 
ergy) of excitons in Ge nanocrystals. This energy does not depend on the samples (Nos. 1, 2, and 3) and is about 7 $\mathrm{meV}$. Since the binding energy of excitons is $4.7 \mathrm{meV}$ in the bulk diamond structure $\mathrm{Ge},{ }^{8}$ the significant enhancement of the binding energy of excitons due to quantum confinement effects ${ }^{9}$ is not observed in Ge nanocrystals. On the other hand, if $\tau$ and $I_{\mathrm{PL}}$ are controlled by the nonradiative processes, the above experimental results mean that the nonradiative recombination rate scarcely depends on the temperature. Since the nonradiative recombination rate strongly depends on the temperature in bulk Ge, the nonradiative processes in Ge nanocrystals are entirely different from those in bulk Ge. Therefore, we consider that the radiative and nonradiative processes in Ge nanocrystals are very complicated. The insensitivity of the PL intensity and the PL spectrum to temperature suggests that Ge nanocrystals in the $<4 \mathrm{~nm}$ size regime resemble isolated molecules rather than an indirect-gap semiconductor. ${ }^{10}$

Now we discuss the mechanism of the roomtemperature photoluminescence from Ge nanocrystals. An important issue is the role of momentum conservation in optical transitions in the indirect-gap Ge. Bulk crystalline Ge has a conduction-band minimum at the $L$ point and the valence-band maximum at the $\Gamma$ point in the Brillouin zone. It is expected that the blue shift of the $L-\Gamma$ indirect transition occurs as size decreases. Takagahara and Takeda ${ }^{9}$ calculated the quantum confinement effect on the $L-\Gamma$ radiative transition using the effective mass approximation and the Luttinger Hamiltonian in Ge quantum dots. They showed that the blue shift of the peak of PL spectrum from infrared to visible region occurs as the diameter decreases: The Ge quantum dots of 14.4, 6.0, and $4.2 \mathrm{~nm}$ in diameter show the peaks of $0.9,1.3$, and $2.2 \mathrm{eV}$ in PL spectra, respectively. The radiative lifetime varies from microseconds to nanoseconds. However, the peak energy of the PL spectrum, the PL lifetime, and the activation energy of the PL intensity do not depend on the average diameter of $\mathrm{Ge}$ nanocrystals. Therefore, our experimental results do not favor the model of the visible luminescence due to the confinement-induced blue shift of the $L-\Gamma$ transition in diamond structure Ge.

On the other hand, our HREM studies show that the crystal structure of Ge having $4 \mathrm{~nm}$ or less in $\mathrm{SiO}_{2}$ glassy matrix does not favor the diamond structure, as previously reported. ${ }^{11,12}$ Saito ${ }^{11}$ pointed out that smaller Ge nanocrystals have a new structure of tetragonal system $(a=0.537$ $\mathrm{nm}$ and $c=0.904 \mathrm{~nm}$ ). The observed spacing of $0.298 \mathrm{~nm}$ as shown in Fig. 2 is nearly equal to $0.292 \mathrm{~nm}$ spacing consisting of the (112) plane of the tetragonal unit. However, at the present stage, we cannot identify a new structure of Ge nanocrystals in $\mathrm{SiO}_{2}$ glassy matrix, because we cannot obtain the electron diffraction pattern of Ge nanocrystals with diameter $\leqslant 4 \mathrm{~nm}$ only. We consider that the structure of crystalline Ge changes critically at about $4 \mathrm{~nm}$ in diameter and that the origin of the visible photoluminescence is Ge nanocrystals of $\leqslant 4 \mathrm{~nm}$ in diameter. In very small nanocrystals, the state mixing occurs for different $\mathbf{k}$ states. ${ }^{10}$ The transition acquires some allowed character from the $\mathbf{k}$ state admixture and $\mathbf{k}$ is not a good quantum number. Therefore, we consider that crystalline Ge of diameter $\leqslant 4 \mathrm{~nm}$ in $\mathrm{SiO}_{2}$ glassy matrix has a new nanostructure exhibiting visible luminescence and has the character of direct optical transition due to hybrid electronic properties between the molecular and solid state limits.

In conclusion, we studied the origin and mechanism of visible photoluminescence in Ge nanocrystals in $\mathrm{SiO}_{2}$ matrix. As the diameter of $\mathrm{Ge}$ crystals decreases below about $4 \mathrm{~nm}$, the strong visible photoluminescence is observed and the nanocrystal structure seems to differ from the diamond structure Ge. We consider that visible photoluminescence of Ge nanocrystals in $\mathrm{SiO}_{2}$ matrix arises from Ge nanocrystals of diameter $\leqslant 4 \mathrm{~nm}$ with a new structure and a molecularlike character. The small Ge quantum dot probably has a character of direct optical transition.

The authors would like to thank Dr. T. Takagahara for sending their manuscript prior to publication and for fruitful comments.

${ }^{1}$ See, for example, L. Brus, IEEE J. Quantum Electron. QE-22, 1909 (1986); L. Brus, Appl. Phys. A 53, 465 (1991); and references therein. ${ }^{2}$ See, for example, E. Hanamura, Phys. Rev. B 38, 1288 (1988); Y. Kayanuma, Phys. Rev. B 38, 9772 (1988); and references therein.

${ }^{3}$ S. Furukawa and T. Miyasato, Phys. Rev. B 38, 5726 (1988).

${ }^{4}$ A. G. Cullis and L. T. Canham, Nature 353, 335 (1991).

${ }^{5}$ Y. Maeda, N. Tsukamoto, Y. Yazawa, Y. Kanemitsu, and Y. Masumoto, Appl. Phys. Lett. 59, 3168 (1991).

${ }^{6} \mathrm{See}$, for example, Abstract Book of Fall Meeting of the Materials Research Society, Symposium AA (Boston, Dec. 1991); Symposium on Luminescence from Indirect Semiconductors, the 1992 March Meeting of the American Physical Society, Indianapolis, 1992.

${ }^{7}$ See, for example, K. Tsunetomo, A. Kawabuchi, H. Kitayama, Y. Osaka, and H. Nasu, Jpn. J. Appl. Phys. 29, 2481 (1990).

${ }^{8}$ C. D. Jeffries, Science 189, 955 (1975); and references therein.

${ }^{9}$ T. Takagahara and K. Takeda, Phys. Rev. B (in press).

${ }^{10}$ R. Rossetti, R. Hull, J. M. Gibgon, and L. E. Brus, J. Chem. Phys. 83, 1409 (1985); Y. Wang, A. Suna, W. Mahler, and R. Kasowski, J. Chem. Phys. 87, 7315 (1987).

${ }^{11}$ Y. Saito, J. Cryst. Growth 47, 61 (1979).

${ }^{12}$ S. Hayashi and H. Abe, Jpn. J. Appl. Phys. 23, L824 (1984). 